\title{
Conséquences des quantités de lait et d'aliment concentré consommées avant sevrage sur les performances de bovins mâles entiers de 3 mois à l'abattage
}

\author{
P. LE NEINDRE et G. PECATTE \\ INRA, Laboratoire de l'élevage bovin \\ Centre de Recherches de Clermont-Ferrand-Theix, \\ F 63122 Ceyrat \\ INRA, Domaine expérimental du Pin \\ Productions animales, Le Pin au Haras, F 61310 Exmes
}

\section{Résumé}

Des bovins mâles de race Normande $(\mathrm{N}=84)$ ont été étudiés de l'âge de 2 semaines à l'abattage. Jusquà 9 mois, ils ont tété deux fois par jour des vaches Normandes. Entre 3 et 9 mois, ils étaient répartis en 3 lots et recevaient de l'herbe ad libitum. Les animaux du premier lot (lot l) tétaient à 2 par vache - soit $8,2 \mathrm{~kg}$ de lait bu par veau et par jour - les autres à 4 par vache - soit $4,4 \mathrm{~kg}$ par jour. Parmi ces derniers, la moitié des animaux n'avait pas d'aliment supplémentairc (lot II), tandis que les autres recevaient $1.4 \mathrm{~kg}$ d'aliment concentré par jour (lot III). A 9 mois, certains animaux ont été abattus, les autres ont été sevrés et ont reçu un même régime à base d'ensilage de maïs jusqu'à l'abattage qui a eu lieu à $550 \mathrm{~kg}$ de poids vif pour une moitié de l'effectif et $600 \mathrm{~kg}$ pour l'autre moitié.

Chaque kilogramme de lait et d'aliment concentré supplémentaires ont permis d'accroître le gain de poids vif entre 3 et 9 mois de respectivement 77 et $186 \mathrm{~g}$ et ont entraîné une diminution de la quantité d'herbe ingérée de 150 et $550 \mathrm{~g}$.

L’efficacité de l’énergie métabolisable du lait paraît plus importante que celles de l’herbe et de l'aliment concentré. Les compositions corporelles des carcasses des animaux des 3 lots abattus au sevrage (4 ou 8 par lot) n'étaient pas différentes. Le poids du rumen des veaux du lot I était. relativement au poids vif, inférieur à celui des animaux des autres lots.

Durant les 3 semaines suivant le sevrage, la croissance des veaux du lot III a été supérieure à cclle des autres animaux. Du sevrage à l'abattage, les animaux du lot III ont eu une croissance significativement supérieure à ceux du lot I. Les taureaux du lot II avaient une croissance intermédiaire. Les efficacités alimentaires ont été légèrement différentes entre lots : supérieures pour les animaux du lot II et inféricures pour ceux du lot I. A même poids à l'abattage, les compositions des carcasses ont été identiques dans les 3 lots.

Mots clés: Taureau, herbe, lait, concentré, allaitement.

\section{Introduction}

Dans une première étude (Le Neindre et al., 1976a) nous avions analysé l'influence des quantités de lait ingérées sur les performances des veaux au pis sevrés à 9 mois. La croissance des veaux qui recevaient le plus de lait avait été supérieure à 
celle des autres veaux. Comme Le Du \& BAKER (1979), nous avons pu montrer qu'une faible ingestion de lait était compensée par une consommation supérieure d'herbe. La qualité de l'herbe est également un facteur important pour expliquer les variations de croissance des veaux (VICINI et al., 1982). Cependant, ces facteurs sont parfois difficiles à maîtriser alors qu'il est possible de distribuer un aliment concentré avant le sevrage afin d'améliorer la croissance des veaux et d'éviter les déficits en herbe.

Les conséquences de l'alimentation avant le sevrage sur la composition corporelle des animaux et leurs aptitudes à l'engraissement intensif en taurillon ont été jusqu'alors très peu étudiées. Les études existant dans la bibliographie sont en nombre limité et portent pour la plupart sur des génisses ou des bœufs de races anglo-saxonnes. La connaissance de ces phénomènes est pourtant très importante à la fois pour l'éleveur et pour l'engraisseur.

Dans l'essai rapporté ici, des veaux mâles ont reçu avant sevrage des quantités de lait et d'aliment concentré différentes et leurs performances ont été comparées jusqu'à l'abattage à environ 15 mois.

\section{Matériel et méthodes}

\section{A. Animaux et dispositif expérimental}

En 1977 et 1978 respectivement, 48 et 36 veaux mâles entiers de race Normande ont été utilisés. La plupart de ces veaux ont été achetés à l'âge de 2 à 3 semaines et affectés le plus tôt possible après le vêlage à des vaches Normandes multipares. Ils ont été élevés au pis jusqu'à 9 mois. Jusqu'à 3 mois environ ils tétaient, à raison de trois veaux par vache, deux fois par jour. Par la suite, trois lots de taille et de caractéristiques identiques (poids, âge) ont été constitués (Schéma 1): les veaux ont reçu beaucoup (Lot I) ou peu de lait (Lots II et III) par le biais du nombre de veaux allaités par vache ( 2 ou 4 ) ; tous recevaient de l'herbe coupée et ceux du lot III ont reçu en plus un aliment concentré.

Après le sevrage, fin octobre et jusqu'à l'abattage, les animaux ont reçu la même ration.

La première année, 4 animaux représentatifs de chaque lot ont été abattus au sevrage. Nous avons complété les résultats d'abattage de ces 12 veaux par ceux de 8 veaux croisés Charolais-Normands provenant d'un autre essai et qui avaient reçu beaucoup ou peu de lait avant l'abattage suivant un protocole identique à celui des lots I et II.

Deux taurillons malades ont été éliminés pendant la période d'engraissement ; ils ne sont donc pas compris dans les calculs. Les deux années, en fin d'engraissement, chaque lot a été divisé en deux groupes identiques dont les animaux ont été abattus aux poids vifs moyens de 550 et $600 \mathrm{~kg}$.

\section{B. Mode de conduite}

\section{Avant le sevrage}

Jusqu'au sevrage, les veaux sont restés en stabulation libre et ont tété les vaches sous surveillance à 8 et 18 heures. Avant la première distribution d'herbe (4 et 8 mai 
respectivement la première et la seconde année) ils avaient à leur disposition du foin et un aliment concentré à volonté. Au-delà et jusqu'au sevrage (19 et 24 octobre respectivement la première et la seconde année), l'herbe qui leur était distribuée était essentiellement du ray-grass anglais. Elle était fauchée chaque jour et distribuée deux fois par jour en quantité telle qu'il devait rester de 10 à $15 \%$ de refus. Les veaux étaient affouragés par case : 2 cases par lot en 1977, deux cases pour le lot I et une seule pour chacun des deux autres lots en 1978. L'aliment concentré (maïs : $75 \%$; soja : $22 \%$; C.M.V. : $3 \%$ ) consommé par les veaux du lot III était distribué en quantité limitée et ajusté chaque semaine de telle façon qu'elle représente en permanence environ la moitié de la quantité de matière sèche d'herbe ingérée.

\section{Après le sevrage}

Le sevrage a commencé 241 et 254 jours après le début de l'allaitement respectivement en 1977 et 1978 . Pendant une semaine, les veaux ont tété une seule fois par jour et tous ont reçu de l'aliment concentré en complément de l'herbe distribuée à volonté. Ils ont ensuite consommé un régime à base d'ensilage de maïs offert à volonté, complété par du maïs grain (3 $\mathrm{kg}$ la première année, 1,5 puis $2,5 \mathrm{~kg}$ la seconde), du tourteau de soja $(0,8 \mathrm{~kg}$ la première année, $1,0 \mathrm{~kg}$ la seconde) et un complément minéral vitaminé. Le stock d'ensilage étant insuffisant la seconde année, la ration a été complétée par $3 \mathrm{~kg}$ de pulpe déshydratée par jour après la première série d'abattages.

\section{Mesures}

Du vêlage au tarissement, les productions laitières des nourrices ont été mesurées une fois toutes les deux semaines par pesées avant et après la tétée des veaux à 2 repas successifs. Du 20 mai au 19 octobre la première année et du 16 mai au 24 octobre la seconde, 5 jours par semaine, les quantités d'herbe distribuées et leur teneur en matière sèche ont été mesurées à chaque repas, les quantités d'herbe refusées et leur teneur en matière sèche une fois par jour. Environ 1 mois après le début du sevrage, respectivement les 16 et 24 novembre la première et la seconde année, et jusqu'à l'abattage, les quantités d'aliments distribuées et refusées ont été mesurées 5 jours par semaine.

La digestibilité de l'herbe a été mesurée pendant toute la période de distribution sur 6 béliers en cage à bilan alimentés à volonté. Ces mesures ont été réalisées sans interruption la première année et deux semaines sur trois la seconde. Dans ce dernier cas, les valeurs manquantes ont été estimées par intrapolation ou extrapolation suivant le stade végétatif de l'herbe. En outre, la première année, la digestibilité d'une ration composée d'herbe et d'aliment concentré, dans des proportions approximativement identiques à celles de la ration donnée aux veaux du lot III, a été mesurée sur des moutons pendant toute la période de distribution de l'herbe.

Nous avons établi des bilans nutritionnels portant avant sevrage sur l'ensemble de la période de mesure et après sevrage sur la période s'étendant du début des mesures aux premiers abattages. Les apports sous forme de lait et d'aliments solides ont été calculés indépendamment, car les résultats présentés par Vermorel, Bouvier \& GeAY (1980) ont montré qu'il n'existait pas d'interaction entre les digestibilités du lait et de l'herbe.

En ce qui concerne l'énergie, le bilan a été effectué en énergie métabolisable car il était très difficile de définir, avant sevrage, des coefficients d'utilisation de l'énergie 
métabolisable en énergie nette des aliments de la ration. Le mode de calcul de la valeur énergétique des fourrages est présenté dans l'Annexe 1. En ce qui concerne la digestibilité de l'énergie de la ration solide du lot III (herbe + concentré), nous avons établi sur 20 semaines de mesures la relation entre les coefficients d'utilisation digestive de la matière organique (en \%) obtenues sur les moutons qui consommaient de l'herbe seule $(\mathrm{X})$ ou une ration herbe et concentré $(\mathrm{Y})$ en introduisant comme variable explicative supplémentaire la proportion d'aliment concentré dans la ration (C). Celle-ci a varié de 33,2 à $41,6 \%$ indépendamment de la digestibilité de l'herbe seule $(\mathrm{r}=0,06)$ :

$\mathrm{Y}=32,9+0,487 \mathrm{X}+0,209 \mathrm{C} \quad \mathrm{R}=0,90 \quad \mathrm{n}=20$

En ce qui concerne l'azote, le bilan est effectué en Protéines Digestibles dans l'Intestin (PDI) pour le lait et les aliments solides en utilisant les équations proposées par Toullec et al. (1978) et par Demarquilly et al. (1978) et en considérant que les valeurs de ces aliments étaient indépendantes. Les valeurs des PDI limitantes ont été seules retenues.

Les caractéristiques des aliments ingérés après sevrage ont été estimées à partir des analyses chimiques lorsqu'elles existaient ou directement sur des tables de valeurs alimentaires des fourrages (Demarquiliy et al., 1978). riance.

Nous avons testé l'influence des facteurs étudiés par analyse de variance-cova-

\section{Résultats}

\section{A. Croissance des veaux}

1. Avant le sevrage

En période préexpérimentale, la croissance des veaux avait été modérée : $794 \pm 143 \mathrm{~g} / \mathrm{j}$. Elle a été en revanche élevée pendant la période expérimentale (en moyenne $1296 \pm 153 \mathrm{~g}$ /jour). Le poids moyen des veaux est passé de $125 \pm 21$ à $329 \pm 33 \mathrm{~kg}$ du début de la période expérimentale au sevrage (fig. 1c).

Pendant la période expérimentale, les animaux du lot II ont eu une croissance très inférieure $(\mathbf{P}<0,001)$ à celle des deux autres lots, ce qui a entraîné un écart de poids au sevrage de $40 \mathrm{~kg}$ avec le lot III et de $43 \mathrm{~kg}$ avec le lot I (tabl. 1).

Intra lots, la croissance pendant la période expérimentale n'est liée ni à la croissance avant la mise en lots ni au poids à cette mise en lots ; il en résulte que le poids au sevrage est lié au poids à la mise en lots avec un coefficient de régression proche de $1(\mathrm{~b}=0,97)$.

\section{Après le sevrage}

Entre le sevrage et l'abattage, la croissance moyenne a été de $1326 \pm 165 \mathrm{~g}$. Nous avons distingué 3 périodes : la première, d'une durée moyenne de 20 jours comprise entre le sevrage et le début des mesures, est la période de transition. La seconde, entre le début des mesures et les premiers abattages, est la période pour laquelle les 

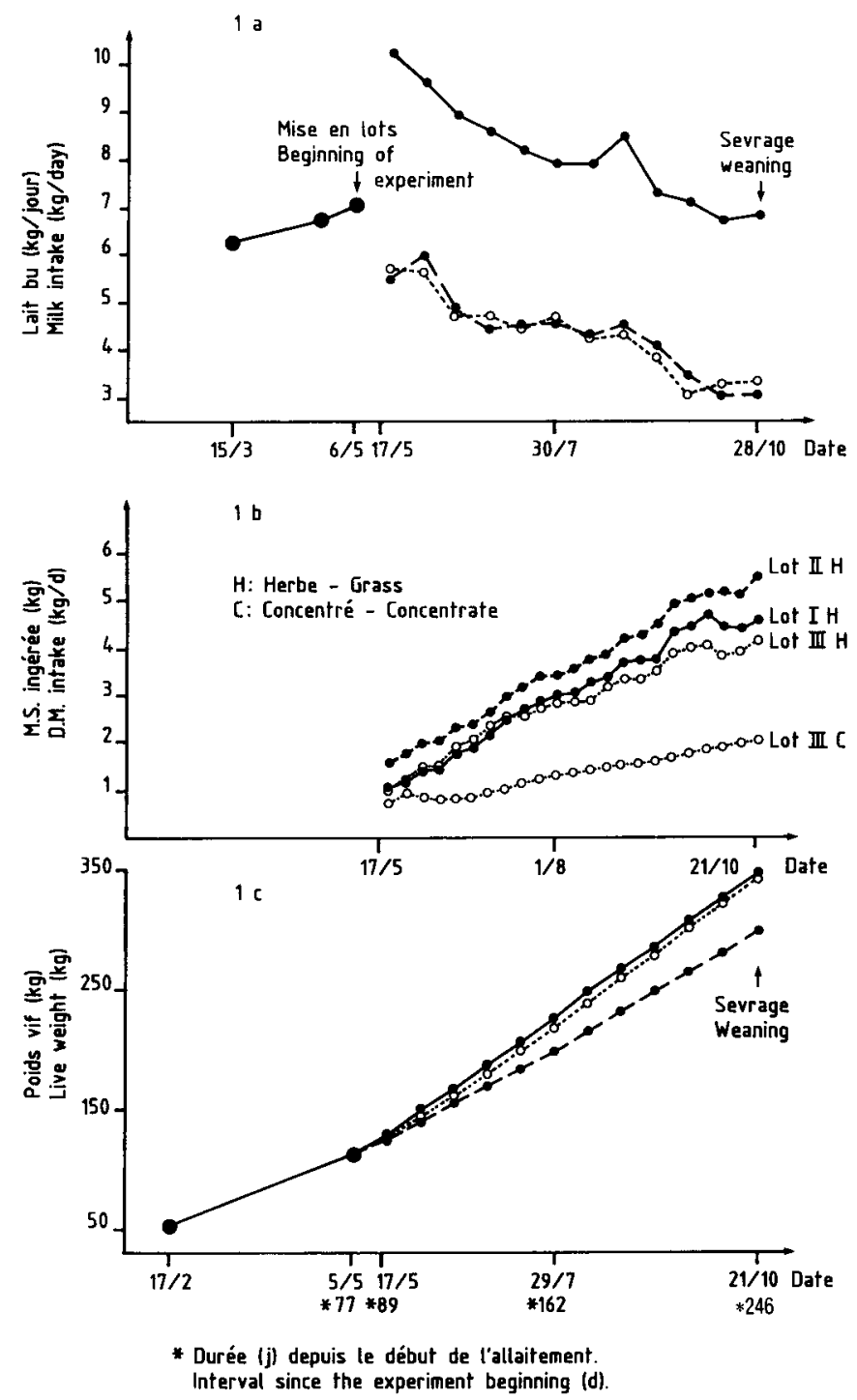

FIG. 1

Influence des quantités de lait (a) et d'aliment concentré (b) ingérées par des veaux sur leur consommation d'herbe (b) et leur croissance (c).

Influence of milk (a) and concentrate (b) intake on calf grass intake (b) and liveweight (c).

Lot I : Quantité de lait bue élevée/High milk intake

Lot II : Quantité de lait bue faible/Low milk intake

Lot III : Quantité de lait bue faible/Low milk intake

+ aliment concentré + concentrate 


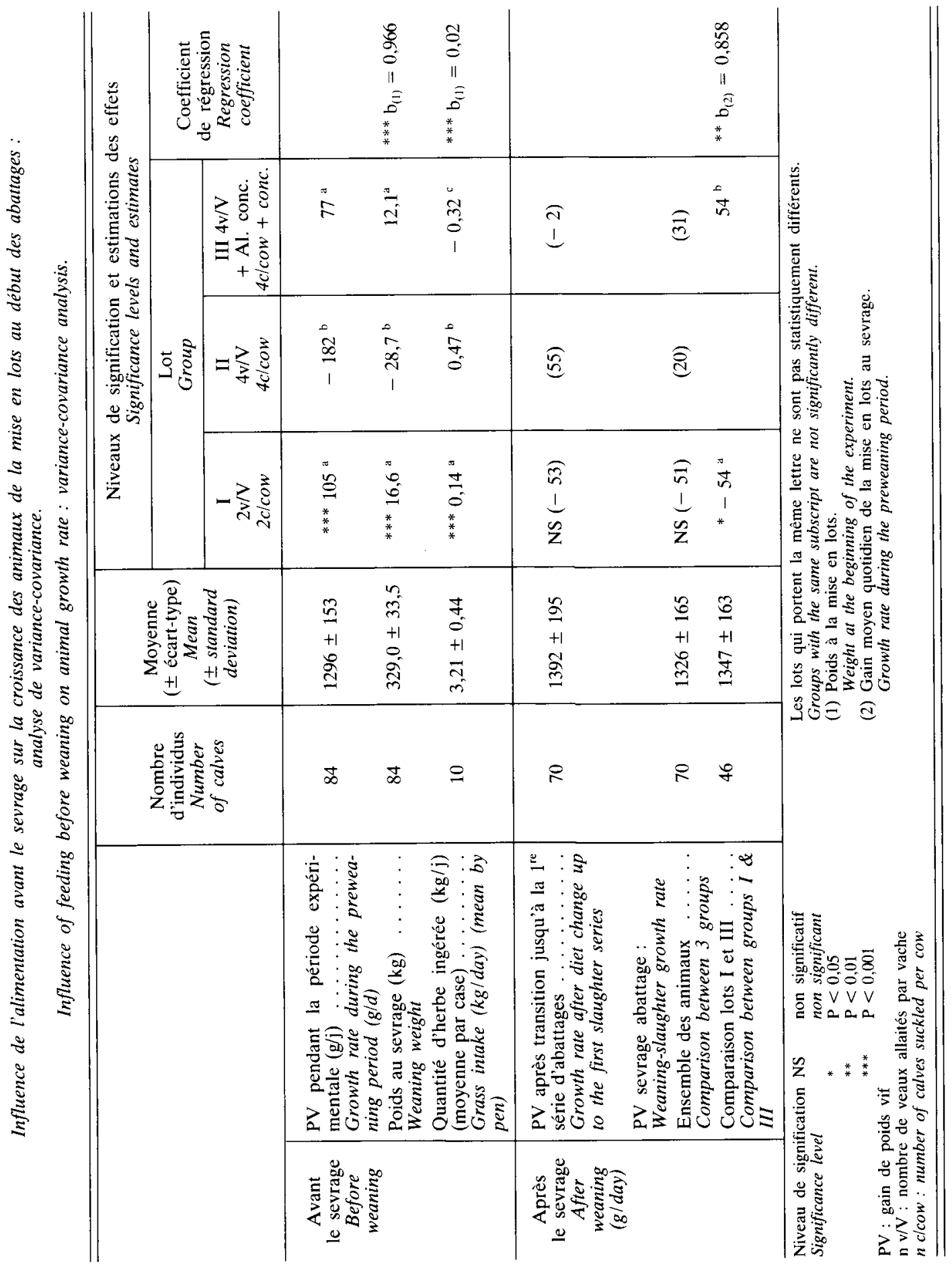


quantités ingérées ont été mesurées. Enfin, la troisième, entre la première et la seconde série d'abattage ne concerne que la moitié des animaux.

Pendant la période de transition, les animaux du lot III ont des croissances très supérieures à celles des deux autres lots (respectivement pour les lots I, II et III : 489, 671 et $1027 \mathrm{~g} / \mathrm{j} ; \mathrm{P}<0,05)$. Par la suite, les différences entre lots sont variables suivant les années. Pendant la seconde période, la croissance des animaux du lot II est de $108 \mathrm{~g} / \mathrm{j}$ supérieure à celle du lot $\mathrm{I}$. Cette différence est à la limite de la signification $(\mathrm{P}=0,055)$. Elle est moins importante si on considère le gain de poids du sevrage à l'abattage $(\triangle=71 \mathrm{~g} / \mathrm{j})$. Afin d'affiner l'analyse, nous avons introduit comme covariable le gain de poids avant le sevrage pour comparer les lots I et III. Les animaux du lot II ayant des gains de poids avant le sevrage très différents de ceux des autres lots n'ont pu être inclus dans cette analyse. Dans ces conditions, les animaux du lot III ont eu une croissance du sevrage à l'abattage significativement supérieure à celle du lot I $(\triangle=108 \mathrm{~g} / \mathrm{j})($ tabl. 1$)$.

\section{B. Quantités d'aliments ingérées}

\section{Avant sevrage}

Les quantités de lait ingérées ont varié du début à la fin de la période expérimentale de 10,2 à $7,0 \mathrm{~kg}$ pour le lot I et de 5,6 à $3,3 \mathrm{~kg}$ en moyenne pour les deux autres lots (fig. 1a) ; soit en moyenne sur toute la période pour les 3 lots respectivement 8,$19 ; 4,48$ et $4,39 \mathrm{~kg}$ par jour (tabl. 2). Les quantités d'aliment concentré distribuées aux animaux du lot III ont augmenté de 0,99 à $1,75 \mathrm{~kg}$ M.S. par jour. Ces consommations de lait et d'aliment concentré ont entraîné des consommations d'herbe très différentes ( $\mathrm{P}<0,001)$ suivant les lots (fig. 1b, tabl. 1).

Le coefficient de substitution du concentré à l'herbe (diminution de la quantité d'herbe ingérée par unité supplémentaire de concentré distribuée) est de 0,55 , très proche de celui estimé à partir des équations du système d'encombrement (JARRIGE et al., 1980): 0,58. Il est bien inférieur à celui obtenu dans cette expérimentation sur les moutons $(0,86)$.

\section{Après sevrage}

Sur l'ensemble de la période, la première année, les quantités totales ingérées ont été identiques entre lots ; la seconde année, les animaux du lot I ont ingéré $500 \mathrm{~g}$ de Matière Sèche (MS) de plus que ceux des autres lots (tabl. 2). Rapportée au poids métabolique, la consommation totale de MS des animaux du lot II a été supérieure à celle des animaux du lot I la première année et identique la seconde année. Quelle que soit l'année, les animaux du lot III ont ingéré moins d'aliment par $\mathrm{kg}$ de poids métabolique que les autres.

\section{Bilan alimentaire}

\section{Avant sevrage}

Le coefficient d'utilisation digestive de la matière organique de l'herbe mesuré sur mouton, a varié entre 78,6 et $72,6 \%$ suivant le stade végétatif de l'herbe et la semaine. Celui de la ration d'herbe et concentré était légèrement plus élevé et a varié entre 79,6 


\begin{tabular}{|c|c|c|c|c|c|c|}
\hline & & & 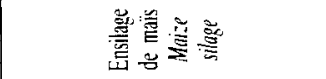 & $\begin{array}{l}\text { 导导导 } \\
\dot{\forall}+\underset{+}{*}\end{array}$ & 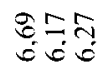 & 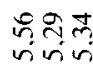 \\
\hline & & 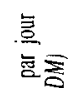 & 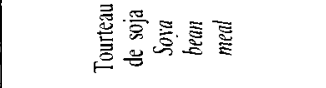 & 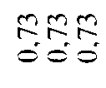 & $\begin{array}{l}\infty \infty \infty \\
\infty \infty \infty \\
\stackrel{\infty}{=} \stackrel{\infty}{=}\end{array}$ & $\begin{array}{l}\mathscr{\infty} \infty \\
\stackrel{\infty}{=}=\end{array}$ \\
\hline & 趈 & 这 & 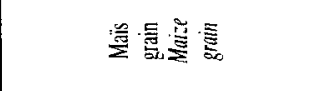 & $\begin{array}{l}\text { nुñ } \\
\text { nini }\end{array}$ & 电昌昌 & $\begin{array}{l}\vec{B} \bar{B} \bar{i} \\
\text { ind }\end{array}$ \\
\hline 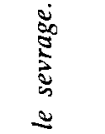 & 竞 & 寒 & 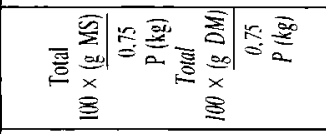 & 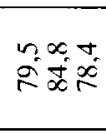 & 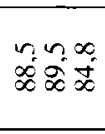 & $\hat{\infty}=\frac{1}{\infty}$ \\
\hline 离 & 舜 & & 悉焉 & $\begin{array}{l}\text { STI } \\
752\end{array}$ & $\begin{array}{l}\hat{\beta} \overline{\bar{n}} \overline{0} \\
\sigma \alpha \infty\end{array}$ & $\begin{array}{l}\infty=0 \\
\infty \infty \infty\end{array}$ \\
\hline 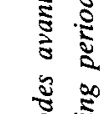 & & & 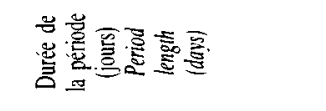 & 哭等 & 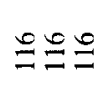 & $\bar{a} \bar{m}$ \\
\hline 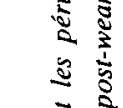 & & & 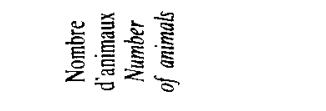 & $=\cong=$ & $\simeq \simeq \simeq$ & Эָন \\
\hline 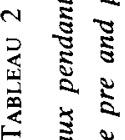 & & & 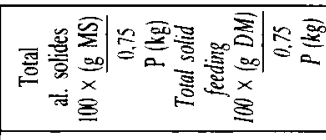 & 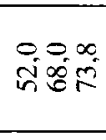 & 范实官 & 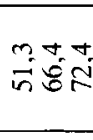 \\
\hline $\begin{array}{l}5 \\
\bar{z} \\
y \\
z \\
z\end{array}$ & & 衰 & 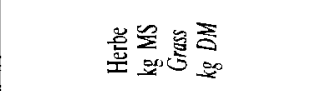 & 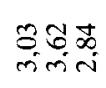 & $\begin{array}{l}\text { जEg } \\
\text { minisi }\end{array}$ & $\begin{array}{l}5 \hat{0} \infty \\
\text { miñ }\end{array}$ \\
\hline 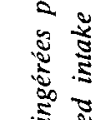 & 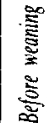 & 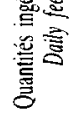 & 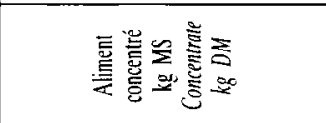 & $11 \stackrel{9}{\stackrel{9}{*}}$ & $1 \mid \stackrel{5}{2}$ & 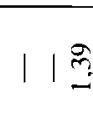 \\
\hline 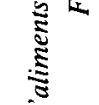 & 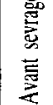 & & 点 & 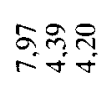 & 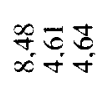 & 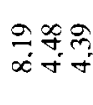 \\
\hline 莺 & & & 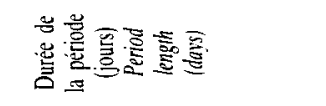 & 㫣员 & $\bar{\Phi} \Xi \bar{\varrho}$ & 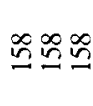 \\
\hline & & & 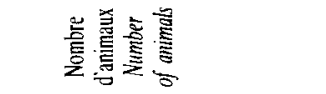 & 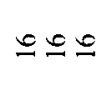 & $\simeq \simeq \cong$ & $\stackrel{\infty}{\sim} \stackrel{\infty}{\sim} \stackrel{\infty}{\sim}$ \\
\hline & & & 호홓ㅎㅁ & ーニミ & ーニミ & ーニミ \\
\hline & & & 毫: & 5 & $\stackrel{\infty}{2}$ & 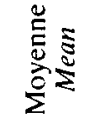 \\
\hline
\end{tabular}




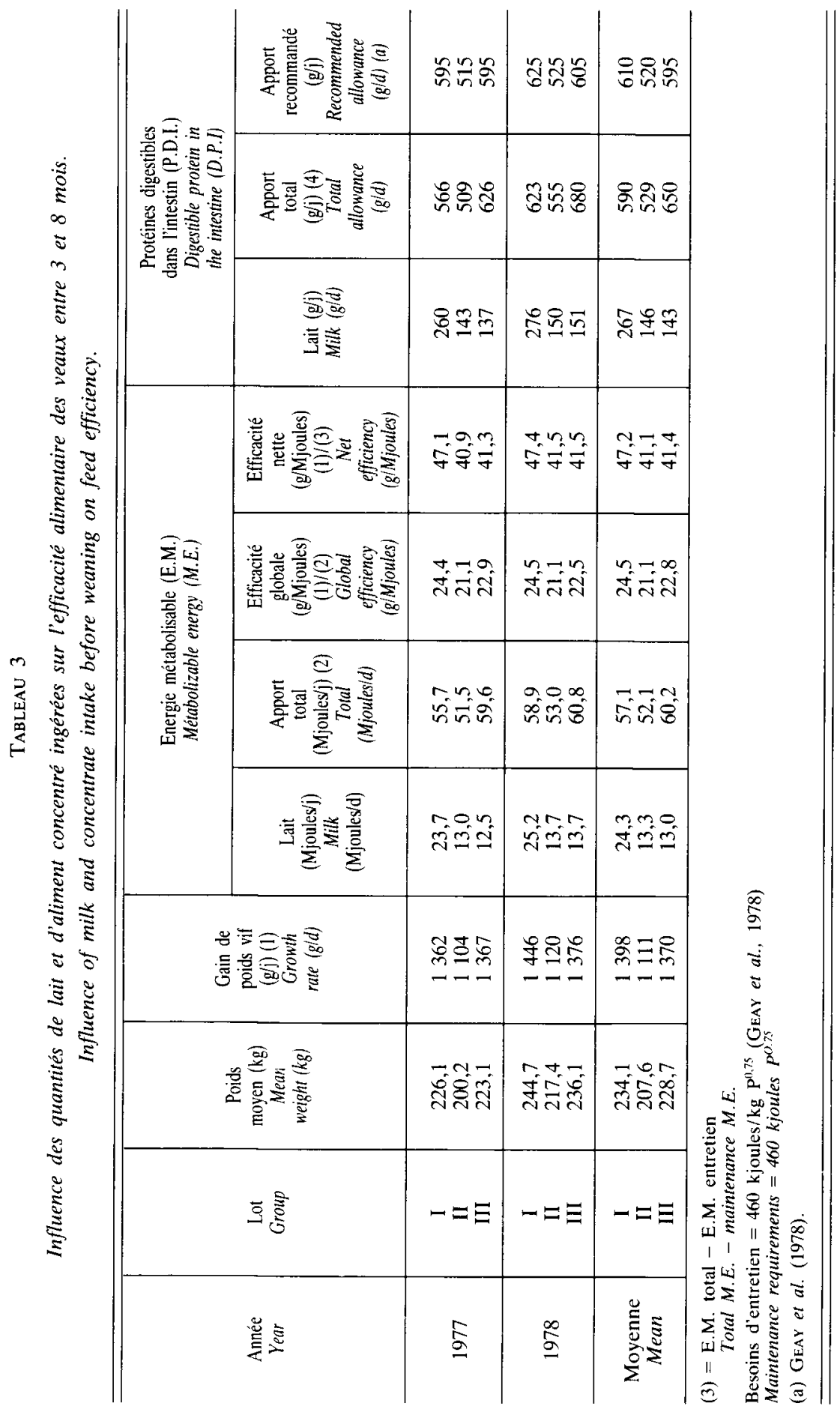


et $74,8 \%$. La teneur en PDI de l'herbe a varié de 128 à $65 \mathrm{~g}$ par $\mathrm{kg}$ de MS et celle de la ration du lot III de 138 à $90 \mathrm{~g}$ par $\mathrm{kg}$ de MS.

Les bilans établis pour les deux années ont été très homogènes. En ce qui concerne l'énergie, l'efficacité globale (gain moyen quotidien/énergie métabolisable totale ingérée) des animaux du lot I a été supérieure à celle des animaux du lot II, le lot III étant intermédiaire. Nous avons ensuite calculé l'efficacité nette (gain moyen quotidien/énergie métabolisable disponible pour la croissance). Celle des animaux du lot I a été supérieure à celles des deux autres lots (tabl. 3).

Les quantités de protéines digestibles dans l'intestin fournies par la ration ont toujours été voisines de celles des besoins calculés, légèrement inférieures pour les veaux recevant beaucoup de lait $(-3 \%)$ et supérieures pour les veaux recevant du concentré $(+9 \%)$.

\section{Après sevrage}

Pour l'énergie, l'efficacité alimentaire globale du lot II a été supérieure à celle du lot $I(+16,3$ et $+7,3 \%$ respectivement la première et la seconde année), celle du lot III étant intermédiaire. Pour calculer l'efficacité nette, les besoins d'entretien ont été pris égaux à $336 \mathrm{Kjoules} \mathrm{d'énergie} \mathrm{nette} \mathrm{par} \mathrm{kg}$ de poids métabolique et les coefficients de transformation de l'énergie métabolisable (EM) en énergie nette pour l'entretien ont été calculés à partir de l'équation suivante : $\mathrm{km}=0,287 \mathrm{EM} / \mathrm{EB}$ (énergie brute) $+0,554$ (VAN Es, 1975). Les différences d'efficacité globale ne sont pas totalement expliquées par une différence des besoins d'entretien puisque les écarts pour l'efficacité nette entre les lots I et II étaient encore de 10,5 et $5,1 \%$ suivant les années. Les apports en protéines digestibles dans l'intestin ont toujours été supérieurs aux besoins calculés (tabl. 4).

\section{Composition anatomique des animaux}

\section{Au sevrage}

Le faible nombre d'animaux du lot III abattus au sevrage $(\mathrm{N}=4)$ ne permet pas une comparaison rigoureuse de ce lot avec les autres. Nous analyserons donc plus spécialement les données concernant les lots I et II ( 8 animaux par groupe). Malgré leurs poids vifs très différents au sevrage $(\triangle=49,8 \mathrm{~kg})$ les veaux des deux groupes ont eu approximativement la même composition de la carcasse et le même poids d'estomacs. En revanche, le poids des intestins a été supérieur chez les veaux qui avaient reçu le plus de lait (tabl. 5).

L'analyse des mensurations des veaux au sevrage fait apparaître que 1) les veaux du lot II étaient plus petits que les autres dans toutes leurs dimensions, 2) les veaux des lots I et III étaient différents : pour le même poids, les premiers étaient plus grands, plus longs mais un peu moins épais que les seconds (tabl. 6).

\section{En fin d'engraissement}

Les poids moyens des animaux en fin d'engraissement de même que la composition de la carcasse ont été effectivement identiques entre lots. Seul, le poids de la caillette des animaux du lot I est apparu significativement supérieur à celles des autres lots. 


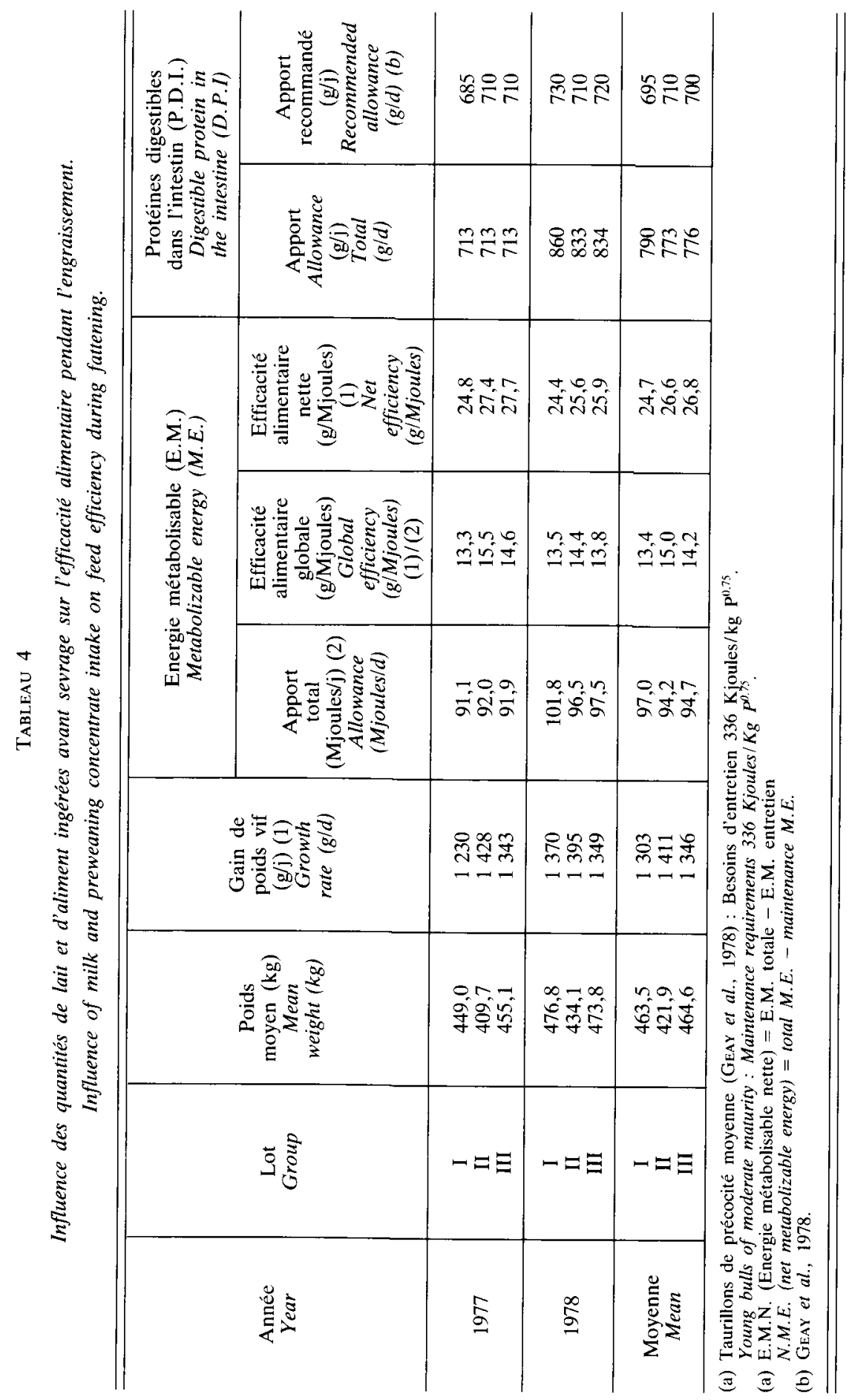




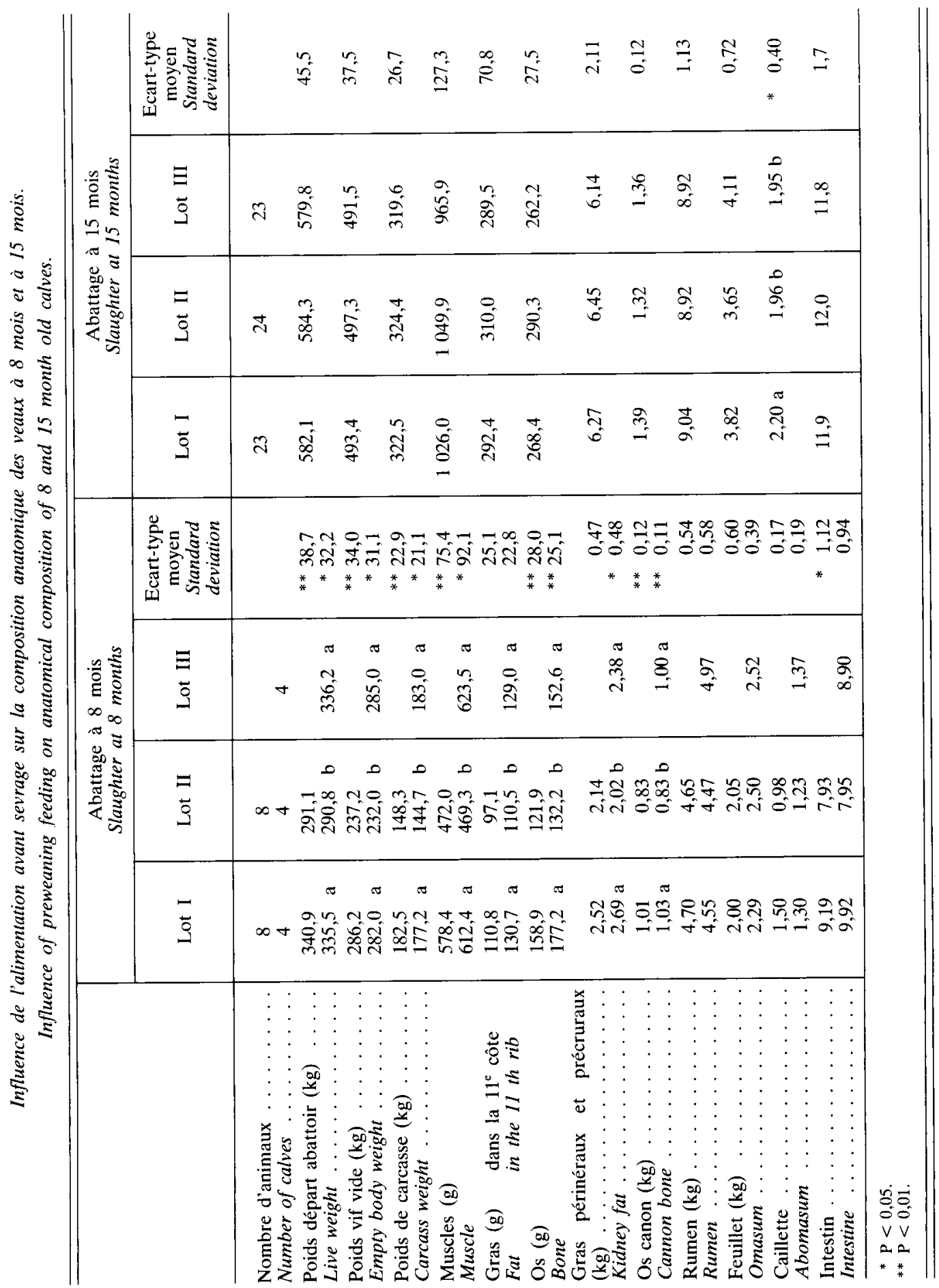




\section{Tableau 6}

Développement corporel des veaux au sevrage.

Body development of suckling calves at weaning.

\begin{tabular}{|c|c|c|c|c|}
\hline Lot & I & II & III & \\
\hline $\begin{array}{l}\text { Nombre de veaux } \ldots . . \\
\text { Number of calves }\end{array}$ & 12 & 12 & 12 & \\
\hline $\begin{array}{l}\text { Poids (kg) } \ldots \ldots \ldots \ldots \\
\text { Liveweight }\end{array}$ & $363,1 \pm 31,2$ & $319,4 \underset{b}{ \pm} 16,0$ & $\begin{array}{c}361,7 \pm 33,8 \\
\mathrm{a}\end{array}$ & $* * *$ \\
\hline $\begin{array}{l}\text { Hauteur au garrot }(\mathrm{cm}) \ldots \\
\text { Height at withers }\end{array}$ & $115,9 \pm 2,6$ & $112,6 \pm 2,9$ & $113,7 \underset{b}{ \pm} 2,4$ & $*$ \\
\hline $\begin{array}{l}\text { Longueur totale }(\mathrm{cm}) \quad \ldots \\
\text { Total length }\end{array}$ & $\frac{176,1 \pm 6,3}{a}$ & $167,2 \pm 6,8$ & $169,5 \pm 5,1$ & $* *$ \\
\hline $\begin{array}{l}\text { Largeur aux trochanters } \\
(\mathrm{cm}) \ldots \ldots \ldots \ldots \\
\text { Width at point of hip }\end{array}$ & $28,5 \pm 1,5$ & $25,9 \pm 1,4$ & $29,0 \pm \frac{1,4}{\mathrm{a}}$ & $* * * *$ \\
\hline $\begin{array}{l}\text { Tour de poitrine }(\mathrm{cm}) \\
\text { Girth circumference }\end{array}$ & $\frac{159,8 \pm 6,8}{\mathrm{ab}}$ & $\frac{155,1 \pm 4,3}{a}$ & $162,5 \pm 6,4$ & $*$ \\
\hline
\end{tabular}

$\begin{array}{ll}\text { Niveau de signification } & * \mathrm{P}<0,05 \\ \text { Significance level } & * * \mathrm{P}<0,01 \\ & * * * \mathrm{P}<0,001\end{array}$

Les lots qui portent la même lettre ne sont pas significativement différents. Groups with same subscript are not significantly different.

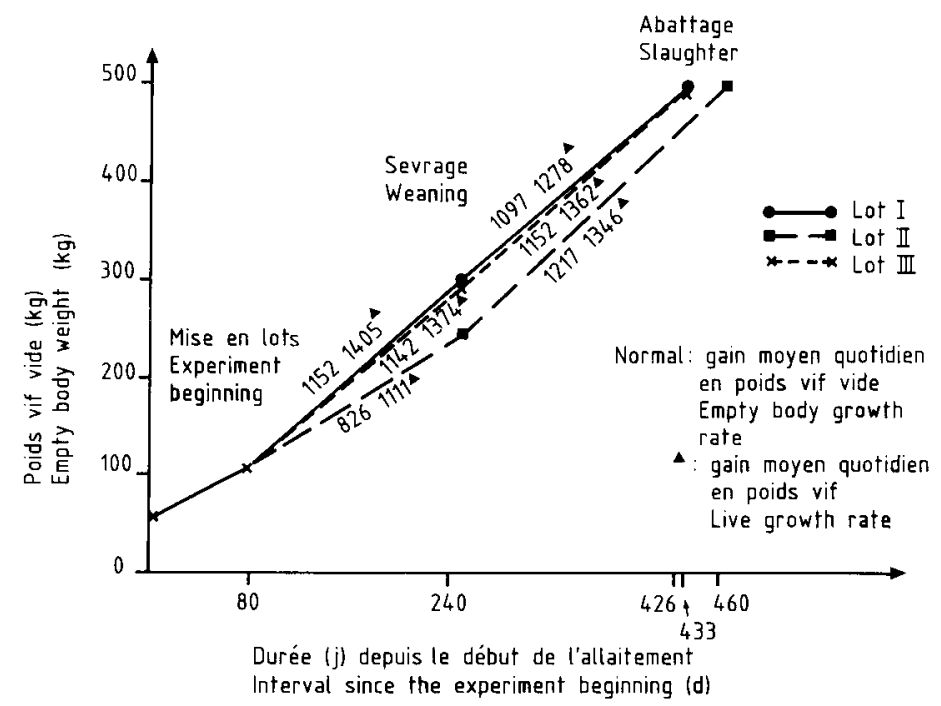

FIG. 2

Poids vif vide de veaux de la mise en nourrice à l'abattage.

Empty body weight of calves from fostering to slaughter. 
Les gains de poids vif vide ont été estimés pour les différentes périodes. Pour cela, les proportions du poids vif vide sur le poids vif des animaux ont été prises égales à $88 \%$ à la mise en lot à 3 mois (MAtHieu, résultats non publiés) et identiques au sevrage à celles obtenues sur les animaux abattus à cette période. Dans ces conditions, avant sevrage, les rapports de croissance entre le lot II et le lot I, sont encore plus importants en poids vif vide $(1,39)$ qu'en poids vif $(1,26)$. Après sevrage ils sont également légèrement différents $(0,95$ en poids vif et 0,90 en poids vif vide) (fig. 2 ).

Rapportés au poids vif vide, les bilans alimentaires se trouvent également modifiés de façon importante. Avant le sevrage, on obtient pour les 3 lots des efficacités nettes de 38,$8 ; 30,6 ; 34,5 \mathrm{~g}$ /MJoules. Le lot I apparaît toujours supérieur au lot II mais dans ces conditions, la valeur du lot III est intermédiaire.

\section{Discussion}

\section{A. Période avant sevrage}

\section{Influence de la quantité de lait bue}

Les quantités de lait et d'aliment concentré supplémentaire ingérées par les veaux ont eu une influence importante sur le gain de poids, la consommation d'herbe et leur composition anatomique au sevrage.

L'influence de la quantité de lait sur la croissance a été mise en évidence dans de nombreuses publications, soit par l'étude de la liaison entre les deux variables à l'intérieur de populations (par exemple : Brumby, Walker \& Gallagher, 1963 ; Melton et al., 1967 ; Poujardieu, 1969 ; Le Neindre et al., 1976b), soit par la comparaison de lots expérimentaux dans lesquels les animaux recevaient des quantités de lait différentes. Ces derniers résultats peuvent seuls être comparés aux nôtres (tabl. 7). Ils sont très variables, l'efficacité du $\mathrm{kg}$ de lait supplémentaire étant comprise entre 36 et $70 \mathrm{~g}$ de gain de poids, valeurs inférieures aux nôtres $(77 \mathrm{~g})$. Ceci peut être expliqué par le positionnement de la période de mesure, entre la naissance et le sevrage. Nos travaux excluaient la période de 0 à 3 mois pendant laquelle les veaux utilisent peut-être moins bien le lait, en raison en particulier de leur état sanitaire. La race utilisée (Normande) permet peut-être aussi une meilleure utilisation du lait que celle obtenue pour les races anglo-saxonnes. Enfin, le mode de conduite peut être invoqué : les animaux sont restés constamment à l'intérieur et ont reçu de l'herbe fauchée, ce qui ne leur a probablement pas permis de trier comme au pâturage et donc de compenser par autant d'herbe leur plus faible quantité de lait bue.

Le fait que les jeunes ruminants consomment d'autant plus d'herbe qu'ils disposent de moins de lait est bien connu et a été décrit dans le cas d'un sevrage tardif aussi bien sur des veaux (PertT, 1972; BAKER, Le Du \& BARKer, 1976 ; Le Neindre et al., 1976a ; Lusby, Stephens \& Totusek, 1976; Le Du \& BAKer, 1979) que sur des agneaux (Hodge, 1966; Penning \& GibB, 1979). Comme dans la première étude (LE NeINDRE et al., 1976a), nous avons obtenu une augmentation de $151 \mathrm{~g}$ de MS d'herbe consommés par $\mathrm{kg}$ de lait en moins, soit approximativement la même valeur que celle obtenue par LE Du \& BaKer (1979) (145 g/kg).

Pour les veaux sevrés tardivement comme pour ceux sevrés très tôt les préestomacs se développent d'autant plus que les veaux reçoivent peu de lait (Harrisson, 


\begin{tabular}{|c|c|c|c|c|c|c|c|c|c|c|c|c|c|c|}
\hline & \multicolumn{2}{|c|}{ 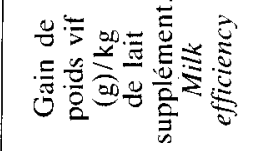 } & $尺$ & 8 & $\hat{0}$ & q & $\approx$ & $\sum_{\infty}^{\infty}$ & P & $\mathcal{F}$ & $\mathscr{f}$ & $\hat{n}$ & $F$ & \\
\hline & \multirow{2}{*}{ 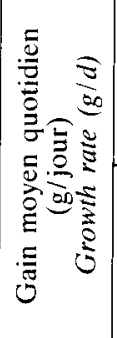 } & 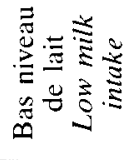 & 导 & $\stackrel{尺}{\Xi}$ & $\underset{E}{i}$ & $\hat{p}$ & $\bar{\Xi}$ & $\underset{\infty}{\stackrel{f}{*}}$ & $\S$ & $\underset{\infty}{\overparen{ఠ}}$ & $\underset{\infty}{\stackrel{F}{(}}$ & $\mathscr{\mathscr { \alpha }}$ & $\leqq$ & \\
\hline \multirow{4}{*}{ 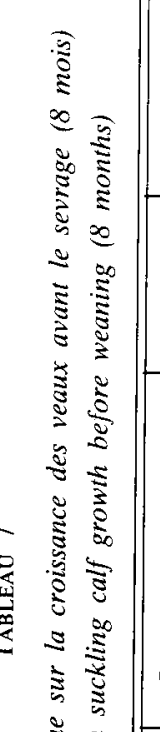 } & & 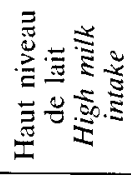 & 䒘 & $\stackrel{\S}{-}$ & $\stackrel{\overparen{S}}{\underline{\Xi}}$ & $\mathscr{\alpha}$ & $\stackrel{\Xi}{\Xi}$ & $\stackrel{?}{\mathrm{~g}}$ & $\supseteqq$ & $\S$ & $\stackrel{\Xi}{\Xi}$ & $\stackrel{\Xi}{\Xi}$ & 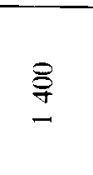 & \\
\hline & \multicolumn{2}{|c|}{ 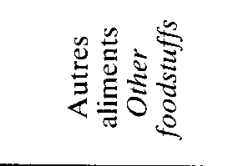 } & \multicolumn{5}{|c|}{ 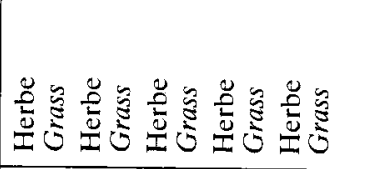 } & $\frac{8}{0}$ & 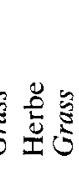 & 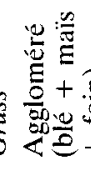 & 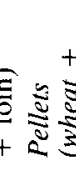 & 胥 & $\frac{1}{5}$ & \\
\hline & \multirow{2}{*}{ 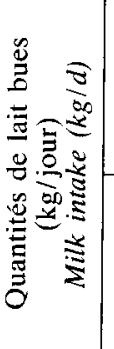 } & 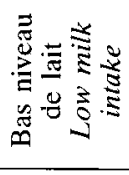 & m & $\stackrel{n}{n}$ & $\stackrel{\infty}{\stackrel{f}{f}}$ & $\hat{c}$ & $\begin{array}{l}\text { in } \\
\text { in }\end{array}$ & $\begin{array}{l}n \\
n\end{array}$ & $\stackrel{\infty}{+}$ & ir & ri & $\vec{\nabla}$ & $\stackrel{n}{\sigma}$ & \\
\hline & & 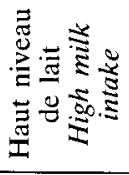 & $r$ & $\stackrel{n}{\varrho}$ & $\vec{\infty}$ & $\stackrel{a}{2}$ & $\stackrel{\Delta}{\sim}$ & $\stackrel{0}{=}$ & $\vec{g}$ & $\stackrel{\infty}{\sigma^{+}}$ & $\stackrel{?}{\varrho}$ & $\stackrel{a}{2}$ & $\stackrel{y}{\infty}$ & \\
\hline 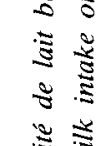 & \multicolumn{2}{|c|}{ 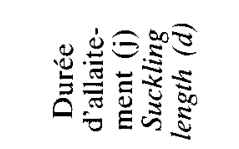 } & $\stackrel{్}{N}$ & $\stackrel{\mathbb{d}}{\stackrel{P}{2}}$ & $\underset{\sim}{\stackrel{P}{7}}$ & 吕 & $\stackrel{*}{\ominus}$ & $\underset{\sim}{\stackrel{9}{4}}$ & 坌 & 孚 & 胥 & 导 & $\stackrel{*}{*}$ & \\
\hline \multirow[t]{2}{*}{ 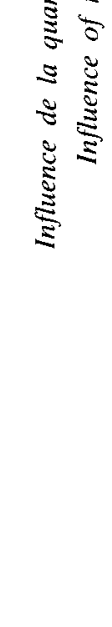 } & \multicolumn{2}{|c|}{ 䢓 } & \multicolumn{2}{|c|}{ 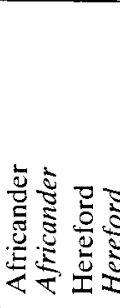 } & 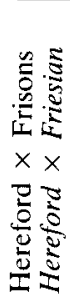 & 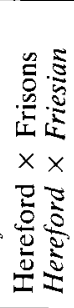 & 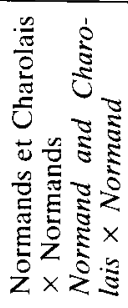 & 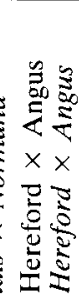 & 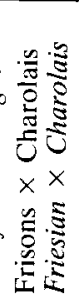 & 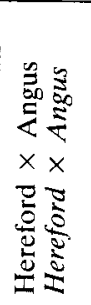 & 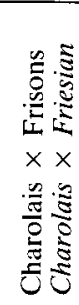 & 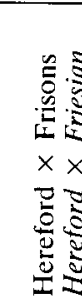 & 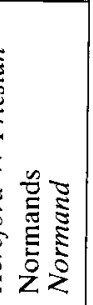 & \\
\hline & & & 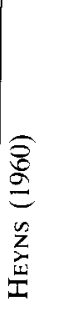 & 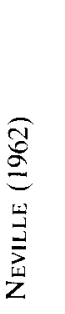 & 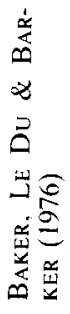 & 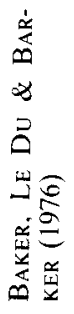 & 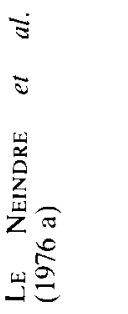 & 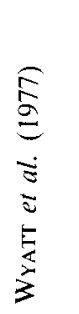 & 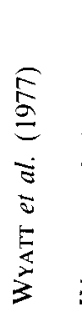 & 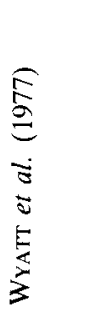 & $\begin{array}{l}\text { E } \\
\text { E } \\
\vdots \\
8 \\
5 \\
5 \\
5 \\
3\end{array}$ & 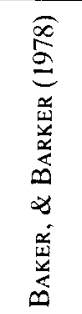 & 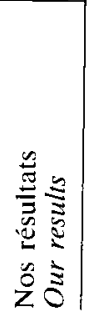 & 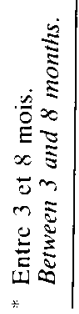 \\
\hline
\end{tabular}


Warner \& LoOsli, 1957 ; Mathieu \& Wegat-Litre, 1961-1962 ; Stobo, Roy \& GasTON, 1966; KAISER, 1976). Cela est vraisemblablement en relation directe avec la présence dans les pré-estomacs d'une plus grande quantité d'aliments solides et de leurs produits de fermentation.

L'efficacité de l'énergie métabolisable du lait paraît être beaucoup plus importante que celle de l'herbe et même que celle du mélange de l'herbe et de l'aliment concentré, du fait probablement de la composition différente des nutriments absorbés. Ces résultats sont en contradiction avec ceux de Vermorel, Bouvier \& GeAY (1980) qui obtiennent une efficacité en énergie nette de la ration supérieure lorsque la quantité de lait offerte diminue, mais dans leur cas, l'aliment solide distribué à volonté est de l'aliment concentré qui permet peut-être aux veaux de mieux compenser la réduction du lait.

\section{Influence de la quantité d'aliment concentré ingérée}

La distribution d'aliment concentré a permis aux animaux du lot III d'avoir la même croissance que ceux du lot I : $405 \mathrm{~g}$ de MS d'aliment concentré ont permis à peu près le même supplément de croissance qu'un kilogramme de lait, soit un gain de $191 \mathrm{~g}$ de poids vif par kilogramme d'aliment concentré. Cette dernière valeur est assez supérieure à celles obtenues dans d'autres expérimentations KuHLMAn, FUrR \& Nelson (1961) 80 à 103 ; Burns, DeEse \& Koger (1966) 164 ; SCARTh et al. (1968) 61 ; Bradley \& Templeton (1973) 115 ; Corah \& Bishop (1975) 104 ; Anthony, Smith \& Grimes (1976) 165 ; Stricker et al. (1979) 102 ; Petit \& Muller (1980) 150. Les races et les types d'animaux utilisés d'une part, les conditions expérimentales d'autre part (pâturage dans beaucoup de cas) expliquent certainement en grande partie la moins bonne efficacité de l'aliment concentré obtenue dans ces expérimentations. La distribution d'aliment concentré a entraîné une diminution importante de l'ingestion d'herbe, inférieure cependant à celle observée sur les moutons.

Bien que le faible nombre d'animaux abattus à 9 mois rende fragile l'interprétation des résultats, on peut noter que la composition de la carcasse des veaux ayant ingéré de l'aliment concentré a été à 8 mois peu différente de celle des animaux des autres lots. Ceci est en contradiction avec les résultats obtenus par d'autres auteurs qui ont observé que les animaux supplémentés étaient un peu plus gras (STUEDEMANN et al., 1968 ; Stricker et al., 1979) ou beaucoup plus gras (Corah \& Bishop, 1975 ; Anthony, Sмith \& Grimes, 1976). Mais les animaux (bouvillons et génisses) avaient, dans ces études, des potentiels de croissance faibles et sans doute une plus grande précocité.

\section{B. Après sevrage}

\section{Influence de la quantité de lait bue}

Différents auteurs ont obtenu des liaisons positives à l'intérieur d'une même population entre les croissances avant et après le sevrage (ReYnolds et al., 1964 : Kennedy \& Henderson, 1975) ou des liaisons nulles (Brinks et al., 1962) ou négatives (Christian, Hauser \& Chapman, 1965 ; Neville et al., 1962 ; Coleman, Pate \& BeARdsley, 1976). Cependant, ces relations peuvent résulter aussi bien de l'alimentation avant le sevrage que des différences interindividuelles des potentiels de croissance qui sont identiques avant et après le sevrage.

Quelques auteurs ont comparé les croissances après sevrage de veaux qui avaient reçu avant sevrage des quantités de lait différentes. La plupart de ces essais ont été 
conduits sur des veaux sevrés précocement et n’ont, généralement, permis de relever aucune influence de l'alimentation lactée avant sevrage sur la croissance après sevrage (Lonsdale \& Tayler, 1969 ; Smith, Callow \& Sweeney, 1973 ; Kaiser \& O'Neill, 1975 ; Kaiser, 1976 ; Brookes \& Davey, 1977 ; Everitt \& Jury, 1977). Seuls, WarDROP (1968) et FOLMAN (1977) observent une meilleure croissance après sevrage des veaux qui ont reçu le plus de lait auparavant. En ce qui concerne les veaux sevrés plus tardivement. Stuedemann et al. (1968) n'observent aucune influence de l'alimentation lactée avant le sevrage sur la croissance ultérieure. En revanche, Brookes \& Hodges (1958) et LE DU \& BAKER (1978) obtiennent des croissances plus élevées lorsque les veaux ont eu moins de lait.

Nos résultats obtenus intra lot confirment la liaison positive entre les croissances avant et après le sevrage. Cependant, l'alimentation n'en est pas la cause puisqu'au contraire, inter lots, les animaux qui avaient reçu le moins de lait et qui étaient les plus légers au sevrage (lot II) ont eu des croissances après sevrage égales ou supérieures à celles des animaux qui en avaient reçu le plus (lot I). En moyenne, entre le sevrage et le tout début des abattages, la différence de poids entre les deux lots s'est réduite d'environ $35 \%$. Ce phénomène de compensation est cependant beaucoup plus marqué la première année, où les quantités totales ingérées en engraissement ont été identiques, que la seconde où les veaux ayant eu le plus de lait ont le plus consommé d'aliment. Cette différence entre les deux années est difficile à expliquer sinon par les qualités différentes des ensilages de maïs distribués : médiocre la première année et bonne la seconde.

\section{Influence de la quantité d'aliment concentré ingérée avant le sevrage}

Suivant les auteurs, les animaux qui ont ingéré de l'aliment concentré avant sevrage ont après sevrage des croissances supérieures (SCARTH et al., 1968) ou inférieures (Stuedemann et al., 1978) à celles de leurs homologues.

Dans notre expérimentation, comme dans celle de Hunsley, Vetter \& Burroughs (1967) les veaux qui avaient reçu du concentré avant le sevrage ont eu une croissance pendant la période de transition supérieure à celle des autres lots.

\section{Conclusion}

Ces expérimentations ont permis d'analyser l'influence jusqu'au sevrage puis jusqu'à l'abattage de deux des facteurs importants de l'alimentation des veaux élevés au pis : les quantités de lait et d'aliment concentré distribuées avant le sevrage. Certaines conclusions paraissent claires, d'autres devront être vérifiées.

Le rôle positif du lait sur la croissance jusqu'au sevrage est confirmé. Comme dans le cas d'un sevrage plus précoce, le veau qui a peu de lait accroît sa consommation d'herbe et développe relativement plus ses pré-estomacs. Cette augmentation de sa capacité d'ingestion est toutefois insuffisante pour compenser une diminution de la quantité de lait distribuée et maintenir la croissance. Le manque de lait peut en revanche être compensé par un apport d'aliment concentré, à raison de $405 \mathrm{~g}$ par $\mathrm{kg}$ de lait en moins. Cet effet reste cependant à préciser en fonction en particulier du niveau de production laitière de la mère, des quantités et de la nature de l'aliment concentré 
distribuées et des quantité et qualité de l'herbe mise à la disposition des veaux, notamment dans les conditions du pâturage.

Les veaux non complémentés qui ont reçu le moins de lait, et qui ont donc eu la croissance la plus faible avant sevrage, ont après sevrage une croissance égale sinon supérieure à celle de leurs homologues. Et à même croissance avant sevrage, les veaux qui ont reçu peu de lait et du concentré ont après sevrage une croissance supérieure à ceux qui ont reçu beaucoup de lait.

A l'abattage à même poids, la composition corporelle est apparue indépendante de l'alimentation avant sevrage. Il semble donc qu'un faible apport de concentré avant le sevrage, pour des animaux à fort potentiel de croissance, puisse être favorable non seulement pendant cette période mais également ne pénalise pas les performances pendant l'engraissement. Il resterait cependant à prouver que des quantités très importantes de lait ou d'aliment concentré n'ont pas alors d'effets négatifs sur les animaux après le sevrage.

Reçu en février 1987.

Accepté en décembre 1987.

\begin{abstract}
Annexe 1
Mode de calcul de la valeur énergétique des rations

Energy content of diets : calculation method
\end{abstract}

- Avant sevrage

Before weaning

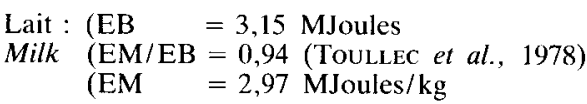

Herbe : équations de Demarquilly et al., 1978 et Vermorel, 1978

Grass : equations from DEMARQUILLY et al., 1978 and VERMOREL, 1978

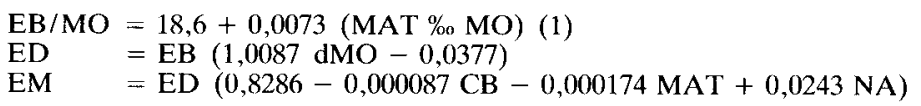

Herbe + concentré

Grass + concentrate

$\mathrm{EB}$ concentré $=18,58$ MJoules $/ \mathrm{kg}$ MS concentrate

EB herbe calculée à partir de l'équation (1) grass from equation (I)

$\mathrm{dMO}($ herbe + al. conc. $)=32,9+0,487$ [dMO herbe seule] grass + concentrate

grass alone

$+20,9\left[\frac{\text { al. conc. }(\mathrm{kg})}{\text { total ingéré }(\mathrm{kg})}\right]$

concentrate/total intake

$\mathrm{ED}=\left[\begin{array}{c}\mathrm{EB} \text { conc. } \\ \text { conc. }+ \text { EB herbe }] \frac{1}{100}[-5,36+1,031 \mathrm{dMO}](\text { SchiEMANN } \text { et al., 1971) }\end{array}\right.$ 
EM (herbe + concentré) calculée à partir de l'équation (2)

(grass + concentrate) from equation (2)

EB : énergie brute (MJoules)

gross energy

ED : énergie digestible (MJoules)

digestible energy

EM : énergie métabolisable (MJoules)

metabolizable energy

MO : matière organique $(\mathrm{kg})$

organic matter

MAT : matières azotées totales ( $\mathrm{g} / \mathrm{kg}$ MS)

total crude protein

MS : matières sèches $(\mathrm{kg})$

dry matter

$\mathrm{CB}$ : cellulose brute ( $\mathrm{g} / \mathrm{kg} \mathrm{MS}$ )

crude fibre

NA : niveau d'alimentation $=1,7$

level of feeding

dMO : digestibilité de la matière organique $(\%)$

organic matter digestibility

- Après sevrage

After weaning

\begin{tabular}{|c|c|c|}
\hline $\begin{array}{l}\text { Teneur en énergie métabolisable des aliments } \\
\text { (MJoules/kg MS) } \\
\text { Metabolizable energy of the foodstuffs }\end{array}$ & 1977 & 1978 \\
\hline $\begin{array}{l}\text { Ensilage de maïs } \\
\text { Maize silage }\end{array}$ & 10,24 & 10,28 \\
\hline $\begin{array}{l}\text { Maiss grain . . . } \\
\text { Maize }\end{array}$ & 14,23 & 14,21 \\
\hline $\begin{array}{l}\text { Tourteau de soja } \\
\text { Soya bean meal }\end{array}$ & 13,84 & 13,84 \\
\hline
\end{tabular}

\section{Summary}

Effect of milk and concentrate intake before weaning on the performance of entire male bulls from three months to slaughter

Eighty-four "Normand" bulls were studied from the age of 2 weeks up to slaughter. Until nine months of age, they were suckled twice a day by Normand cows. From 3 to 9 months of age, they were distributed into three groups (scheme 1) and fed fresh grass ad libitum. Animals from group I were suckled by two per cow $(8.2 \mathrm{~kg}$ milk per animal and per day), whereas in the other groups they were four calves per cow $(4.4 \mathrm{~kg} /$ day $)$. In group II, the animals did not receive any supplementary food, whereas in group III they received $1.4 \mathrm{~kg}$ concentrate per day. At 9 months of age, some animals were slaughtered, the others were weaned and fed a maize silage diet until slaughter. Half the animals were slaughtered at $550 \mathrm{~kg}$ live weight, the other half at $600 \mathrm{~kg}$.

Each supplementary $\mathrm{kg}$ of milk and concentrate led to a live weight gain of 77 and $191 \mathrm{~g}$, respectively in 3-9 month calves and to a reduction in grass intake of 150 and $550 \mathrm{~g}$, respectively (Table 2). 
Efficiency of milk metabolizable energy was higher than that of grass and concentrate (Table 3). Carcass composition of animals from the three groups slaughtered at weaning were not different. In group I rumen weight relative to live weight was lower than that of the other groups (Table 5).

During the three weeks after weaning, growth rate of group III was higher than that of the other groups. From weaning to slaughter, growth rate of group III was significantly higher than that of group I. Young bulls of group II had an intermediate growth rate. Feed efficiency was slightly different between groups : higher in group II and lower in group I. At the same slaughter weight, carcass compositions were similar in the three groups.

Key words : Bull, grass, milk, concentrate, suckling.

\section{Références bibliographiques}

Anthony W.B., Smith L.A., Grimes H.W., 1976. Effects of diet on growth of creep-fed calves. $J$. Anim. Sci., 43 (1) 313 (Abst.).

Baker R.D., Barker J.M., 1978. Milk fed calves. 4. The effect of herbage allowance and milk intake upon herbage intake and performance of grazing calves. J. Agric. Sci. Camb., 90, 3138 .

Baker R.D., Le Du Y.L.P., Barker J.M., 1976. Milk fed calves. The effect of milk intake upon the herbage intake and performance of grazing calves. J. Agric. Sci. Camb., 87, 187-196.

BRADley N.W., Templeton W.C., 1973. Interrelations of season of calving, kind of pasture, and supplemental feeding on nursing calves on traits of Economic Importance in feeder calf production. Univ. of Kentucky Agr. Exp. Stu. Res. Work Unit Prog. Rep., 227, 5-10.

Brinks J.S., Clark R.T., KiefFer N.M., Quesenberry J.R., 1962. Genetic and environmental factors affecting performance traits of Hereford bulls. J. Anim. Sci., 21, 777-781.

Brookes A.J., Hodges J., 1958. Studies in beef production. I. The effect of level of feeding and of breed on the growth and fattening of spring born cattle. J. Agric. Sci., 53, 78-101.

Brookes I.W., Davey A.W.F., 1977. Effect of feeding different levels of whole milk on growth rates of dairy heifers calves before and after weaning. N.Z. J. Agric., Res., 20, 1-5.

Brumby P.J., Walker D.K., Gallagher R.M., 1963. Factors associated with growth in beef cattle. N.Z. J. Agric. Res., 6, 526-537.

Burns W.C., Deese R.E., Koger M., 1966. Creep feeding beef calves in Florida ARS-USDA. Prod. Res. Rep, 88.

Christian L.L., Hauser E.R., Chapman A.B., 1965. Association of preweaning and postweaning traits with weaning weight in cattle. J. Anim. Sci., 24, 652-659.

Coleman S.W., Pate F.M., Beardsley D.W., 1976. Effect of level of supplementation energy fed grazing steers on performance during the pasture and subsequent drylot period. J. Anim. Sci., 42, 27-35.

Corah J.R., Bishop A.H., 1975. Effect of creep feeding oat grain to feed calves on their growth rate, carcass composition and postweaning performance in a feedlot. Aust. J. Exp. Agric. Anim. Husb., 15, 293-307.

Demarquilly C., Andrieu J., Saunant D., Dulphy J.P., 1978. Composition et valeur nutritive des aliments, in JARrige $R$. « Alimentation des Ruminants ». INRA Publications, Route de Saint-Cyr, 78000 Versailles, 469-518.

Everitt G.C., JuRY K.E., 1977. Growth of cattle in relation to nutrition in early life. N.Z. $J$. Agric. Res., 20, 129-137.

Folman Y., 1977. A note on the relationship between live weight gain during calfhood and subsequent performance of intact male calves of the Isreali-Friesian breed. Anim. Prod., 24, 283-286.

Geay Y., Robelin J., Beranger C., Micol D., Gueguen L., Malterre C., 1978. Bovins en croissance et à l'engrais. In JARRIGE R. "Alimentation des Ruminants ". INRA Publications, Route de Saint-Cyr, 78000 Versailles, 297-343. 
Harrisson H.N., Warner R.C., Looslu J.K., 1957. The retrogression of rumen of dairy calves following the substitution of milk for hay grain diet. J. Anim. Sci., 16, 1088-1097.

Heyns H., 1960. The milk production of the dam and growth of the calf. Anim. Breed. Abstr., 29, 1324.

Hodge R.W., 1966. The relative pasture intake of grazing lambs at two levels of milk intake. Aust. J. Exp. Agric. Anim. Husb., 6, 314-16.

Hunsley R.E., Vetter R.L., Burroughs W., 1967. Effects of creep-feeding and diethylstilbestrol implants on preweaning performance of male beef calves. J. Anim. Sci., 26, 1482 (Abst.).

Jarrige R., Beranger C., Demarquilly C., Dulphy J.P., Geay Y., Hoden A., Journet M., Malterre C., Micol D., Pett M., Robelin J., 1980. Le système des unités d'encombrement pour les bovins. Bull. Techn. C.R.Z.V. Theix INRA, 38, 57-79.

KaISER A.G., 1976. The effects of milk feeding on the pre- and post-weaning growth of calves and on stomach development at weaning. J. Agric. Sci. Camb., 87, 357-363.

Kaiser A.G., O'Neill G.H., 1975. Rearing dairy beef calves by multiple suckling. Aust. J. Exp. Agric. Anim. Husb., 15, 314-319.

Kennedy B.W., Henderson C.R., 1975. Genetic, environmental and phenotypic correlations between growth traits of Hereford and Angus calves. Can. J. Anim. Sci., 55, 503-512.

Kuhlman L.R., Furr A.D., Nelson A.B., 1961. Creep feeds and creep feeding fall calves until spring vs weaning. J. Anim. Sci., 20, 399.

LE Du Y.L.P., BaKer R.D., 1978. A note on the effect of the level of milk consumption prior to weaning upon the silage intake and performance of weaned «suckled» calves. Anim. Prod., 27, 117-120.

Le Du Y.L.P., Baker R.D., 1979. Milk fed calves. 5. The effect of a change in milk intake upon the herbage intake and performance of grazing calves. J. Agric. Sci. Camb., 92, 443-447.

Le Neindre P., Petrt M., Muller A., 1976 a. Quantités d'herbe et de lait consommées par des veaux au pis. Ann. Zootech., 25, 521-531.

Le Neindre P., Petit M., Tomassone R., Roux C., 1976 b. Production laitière des vaches allaitantes et croissance de leurs veaux. I. Race Limousine. Ann. Zootech., 25, 221-241.

LONSDAle C.R., TAYLeR J.C., 1969. The artificial rearing of calves and their growth on grass diets. II. The effect of length of period of feeding cold milk substitute to spring-born calves at pasture. J. Agric. Sci. Camb., 73, 483-488.

Lusby K.S., Stephens D.F., Totusek R., 1976. Effects of milk intake by nursing calves on forage intake, on range and creep intake and digestibility in drylot. J. Anim. Sci., 43, 1066-1071.

Mathieu C.M., Wegat-Litre E., 1961-1962. Mise au point d'une méthode d'alimentation des veaux d'élevage. Ann. Zootech., 10, 161-175. Ann. Zootech., 11, 197-207.

Melton A.A., Riggs J.K., Nelson L.A., Cartwright T.C., 1967. Milk production composition and calf gains of Angus, Charolais and Hereford cows. J. Anim. Sci., 26, 804-809.

NEVILLE W.E., 1962. Influence of dam's milk production and other factors on 120 and 240 day weight of Hereford calves. J. Anim. Sci., 21, 315-320.

Neville W.E., David D.M., Mc Campbell H.C., Sell O.E., 1962. Influence of dam's milk production and other factors on postweaning performance and carcass characteristics of Hereford cattle. J. Anim. Sci., 21, 943-949.

Penning P.D., GibB H.J., 1979. The effect of milk intake on the intake of cut and grazed herbage by lambs. Anim. Prod., 29, 53-67.

Petrt M., 1972. Emploi du temps des troupeaux de vaches mères et de leurs veaux sur les pâturages d'altitude de l'Aubrac. Ann. Zootech., 21, 5-27.

Petit M., Muller A., 1980. Utilisation du pâturage par les vaches allaitantes : influence du chargement. Ann. Zootech., 29, 317-338.

Poujardieu B., 1969. Recherche d'une méthode d'estimation de la production laitière des femelles ovines et bovines pendant la phase d'allaitement. Ann. Zootech., 18, 299-315.

Reynolds W.L., De Rouen T.M., High J.W., Temple R.S., 1964. Relationship of preweaning to postweaning performance of fed steers and replacement heifers. J. Anim. Sci., 23, 305 (Abstr.). 
Scarti R.D., Miller R.C., Philipps P.J., Sherritt G.W., Ziegler J.H., 1968. Effects of creep feeding and sex on the rate and composition of growth of crossbred calves. J. Anim. Sci., 27, 596-599.

Schiemann R., Jentsch W., Wittenburg H., 1971. Zur Abhängigkeit der Verdaulichkeit der Energie und der Nährstoffe von der höhe der Futteraufnahme und der Rations zusammenstzung bei Milchkühen. Arch. Tierernähr, 21, 223-240.

Smith M.E., Callow C., Sweeney B.J., 1973. Ten and eighteen week suckling of Friesian steers. Proc. N.Z. Soc. Anim. Prod., 33, 166-175.

Stobo I.J.F., Roy R.M.B., Gaston H.J., 1966. Rumen development in the calf. Br. J. Nutr., 20, 171-215.

Stricker J.A., Matches A.G., Thompson G.B., Jacobs V.E., Martz F.A., Wheaton H.N., Currence H.D., Krause G.F., 1979. Cow-calf production on tall fescue Ladino clover pastures with and without nitrogen fertilization on creep feeding : spring calves. J. Anim. Sci., 48, 13-25.

Stuedemann J.A., Guenther J.J., Ewing S.A., Morrison R.D., Odell G.V., 1968. Effect of nutritional breed level imposed from birth to 8 months of age on subsequent growth and development patterns of full-fed calves. J. Anim. Sci., 27, 234-241.

Toullec R., Thivend P., Vermorel M., Gueguen L., 1978. Veaux. In Jarrige R. « Alimentation des Ruminants ». INRA Publications, Route de Saint-Cyr, 78000 Versailles, 245-296.

VAN Es A.J.H., 1975. Feed evaluation for dairy cows. Livest. Prod. Sci., 2, 95-107.

Vermorel M., 1978. Ch. 2. Energie, in Jarrige R. "Alimentation des Ruminants". INRA Publications, Route de Saint-Cyr, 78000 Versailles, 47-88.

Vermorei. M., Bouvier J.C., Geay Y., 1980. Utilisation de l'énergie des alimerits par le veau ruminant. Influence de l'apport de lait, du niveau d'alimentation et de l'âge du veau. Ann. Zootech., 29 (1), 65-86.

Vicini J.L., Pridge E.C., Bryan W.B., Varga C.A., 1982. Influence of forage species and creep grazing on a cow-calf system. I. Intake and digestibility of forages. J. Anim. Sci., 55, 752.

Wardrop I.D., 1968. Birht weight, liveweight gain in early life and subsequent gain in sheep and cattle. Aust. J. Agric. Res., 19, 837-844.

Wyatt R.D.. Gould M.B., Witheman J.V., Totusek R., 1977. Effect of milk level and biological type on calf growth and performance. J. Anim. Sci., 45, 1138-1145. 